\title{
Bilayer Excitons in Two-Dimensional Nanostructures for Greatly Enhanced Thermoelectric Efficiency
}

\author{
Kai Wu, ${ }^{1, *}$ Louk Rademaker, ${ }^{2}$ and Jan Zaanen ${ }^{3}$ \\ ${ }^{1}$ Stanford Institute for Materials and Energy Sciences, SLAC National Accelerator Laboratory, \\ 2575 Sand Hill Road, Menlo Park, California 94025, USA \\ ${ }^{2}$ Kavli Institute for Theoretical Physics, University of California, Santa Barbara, California 93106, USA \\ ${ }^{3}$ Institute-Lorentz for Theoretical Physics, Leiden University, P.O. Box 9506, Leiden, Netherlands
}

(Received 26 August 2014; published 25 November 2014)

\begin{abstract}
Currently, a major nanotechnological challenge is to design thermoelectric devices that have a high figure of merit. To that end, we propose to use bilayer excitons in two-dimensional nanostructures. Bilayerexciton systems are shown to have an improved thermopower and an enhanced electric counterflow and thermal conductivity, with respect to regular semiconductor-based thermoelectrics. We suggest an experimental realization of a bilayer-exciton thermocouple. Based on current experimental parameters, a bilayer-exciton heterostructure of $p$ - and $n$-doped $\mathrm{Bi}_{2} \mathrm{Te}_{3}$ can enhance the figure of merit an order of magnitude compared to bulk $\mathrm{Bi}_{2} \mathrm{Te}_{3}$. Another material suggestion is to make a bilayer out of electrondoped $\mathrm{SrTiO}_{3}$ and hole-doped $\mathrm{Ca}_{3} \mathrm{Co}_{4} \mathrm{O}_{9}$.
\end{abstract}

DOI: 10.1103/PhysRevApplied.2.054013

\section{INTRODUCTION}

Ranging from household refrigeration to waste heat in power plants, the problem of converting heat into electricity or vice versa is of paramount technological importance [1]. Instead of solving this issue with compression-based heat pumps and the like, solid-state materials might offer a resolution. Among the notable advantages of using solidstate Peltier or Seebeck devices are their light weight, the absence of any moving parts, and the elimination of any environmentally unfriendly substances. However, the main problem remains to devise a thermoelectric device with sufficient efficiency.

A typical thermoelectric device consists of both $p$-type and $n$-type materials, as shown in Fig. 1 . The key to realizing an efficient thermoelectric device is finding materials with high thermoelectric performance, commonly expressed in terms of the dimensionless figure of merit $[2,3]$

$$
z T=\frac{\alpha_{e}^{2} / L}{1+\frac{\kappa_{\mathrm{ph}}}{\kappa_{e}}},
$$

where $\alpha_{e}, \kappa_{\mathrm{ph}}, \kappa_{e}$, and $L$ are the Seebeck coefficient, the phonon thermal conductivity, the thermal conductivity of the electrons and the Lorenz number, respectively. Following the Wiedemann-Franz law, the Lorenz number $L$ is defined by the ratio [4]

$$
L=\frac{\kappa_{e}}{\sigma_{e} T},
$$

where $\sigma_{e}$ is the electrical conductivity and $T$ is the temperature. The theoretical value for the Lorenz number of

\footnotetext{
*kaiwu@stanford.edu
}

degenerate metals is given by $L_{0}=\frac{\pi^{2}}{3}\left(\frac{k_{B}}{e}\right)^{2}=2.44 \times$ $10^{-8} \mathrm{~V}^{2} \mathrm{~K}^{-2}[5,6]$. Indeed, experimentally measured values of $L$ show only small deviations from this Sommerfeld value, namely, $L=(1.7-2.6) \times 10^{-8} \mathrm{~V}^{2} \mathrm{~K}^{-2}$ in elemental metals, metallic compounds, and semiconductors [7-10].

Therefore, the obvious strategy to maximize the thermoelectric figure of merit is by increasing the thermopower $\alpha$ and decreasing the phonon thermal conductivity $\kappa_{\mathrm{ph}}$. Indeed, the central maxim of most thermoelectric research is summarized by the phrase "phonon-glass electroncrystal" $[1,11,12]$. A different route to increase $z T$, however, is to involve low-dimensional nanostructures $[13,14]$. Specifically, the use of 2D quantum wells [15] enhances the bulk value of $z T$ for the "state-of-the-art" hole-doped semiconductor $\mathrm{Bi}_{2} \mathrm{Te}_{3}$ from its bulk value of $z T=1$ [16] to $z T \sim 2.4$ for thin films [17]. In a 2D electron gas of doped $\mathrm{SrTiO}_{3}$, a $z T \sim 2.4$ has been achieved [18]. However, to overcome the competition of conventional heat pumps regarding efficiency, we need to find new materials to enhance $z T$ to 4 or greater $[1,19]$. In this paper, we present a concept that has the potential to increase the figure of merit $z T$ by a factor of 60 . For this, we need to mobilize bilayer excitons.

An exciton is the bound state of an electron and a hole. In a bulk material, the electron and hole can recombine and thus annihilate the exciton. In order to avoid this annihilation, it has been proposed to spatially separate the electrons and holes in two different layers [20,21]. Such a heterostructure of a $p$-type and $n$-type material can support bilayer excitons. Since the bound state of an electron and a hole is effectively a boson, it can undergo Bose-Einstein condensation, and substantial experimental and theoretical 


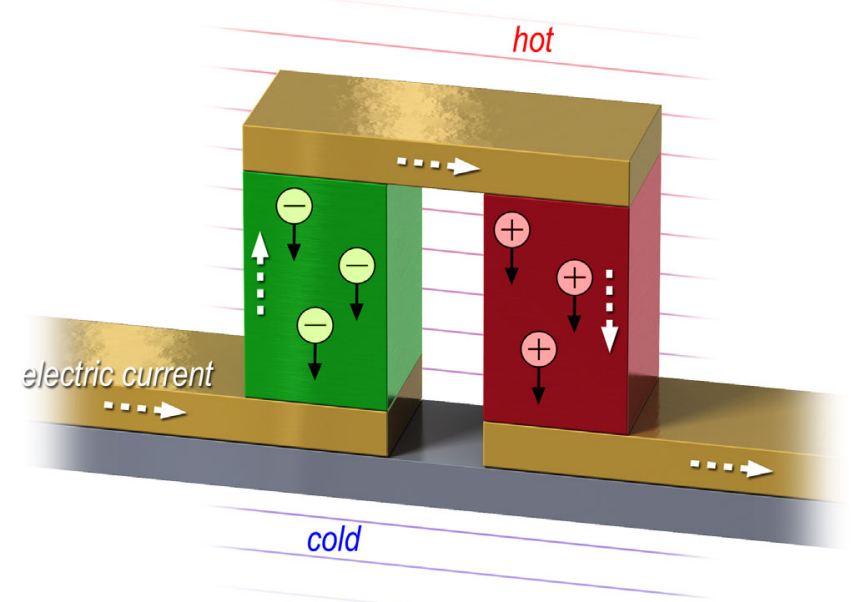

FIG. 1. Traditional thermocouple. A traditional thermocouple device consists of an $n$-type material (in green) and a $p$-type material (in red) connected electrically in series but parallel with respect to the temperature gradient. The charge carriers move from the hot end to the cold end (black arrows), generating an electrical current (white dashed arrows).

efforts are devoted to the realization of a bilayer-exciton condensate [22-27].

The bilayer-exciton system displays a remarkable similarity to a thermoelectric couple, as shown in Fig. 1. Such a device consists of a $p$-type and $n$-type thermoelectric material connected in series [3]. A heat gradient will cause the charge carriers to diffuse from the hot side to the cold side, thus converting the heat gradient into an electrical current. Now imagine that one brings the $p$ - and $n$-type materials close to each other. At distances of the order of nanometers, the Coulomb attraction between the electrons and holes can bind them together into bilayer excitons. As shown in Fig. 2, the application of a heat gradient causes these excitons to flow from the hot side to the cold side. Even though excitons are charge neutral, the fact that the holes and electrons are spatially separated implies that an exciton current amounts to two opposite currents in the two layers [28]. If one closes the circuit in the counterflow setup (see Fig. 2), the exciton current is converted into an electrical current. Such excitonic counterflow conductivity has been demonstrated experimentally [29].

Though most bilayer-exciton research focuses on the Bose condensation of excitons, for thermoelectric applications uncondensed excitons are required. Although the counterflow conductivity of the exciton Bose condensate is infinite due to the superfluidity, the Seebeck coefficient vanishes faster and the figure of merit drops to zero [30]. However, we show here that the high-temperature properties of a gas of bilayer excitons will lead to an exceptionally large thermopower, combined with a small Lorenz number. In addition, the remarkably weak coupling between excitons and phonons, the dominant scattering mechanism at high

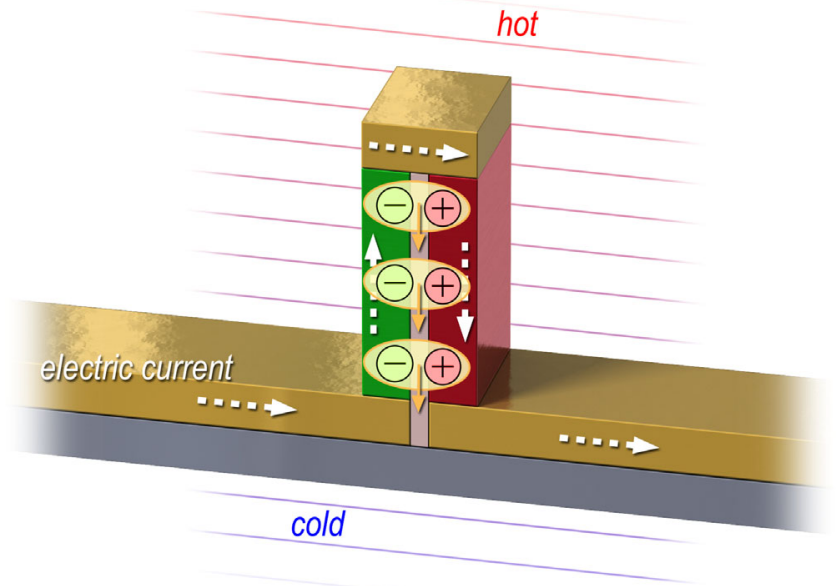

FIG. 2. Bilayer-exciton thermocouple. In a bilayer-exciton thermocouple, as presented in this paper, the $p$ - and $n$-type materials are brought close together so that bilayer excitons can form. In the thermoelectric device, the excitons move from the hot side to the cold side (orange arrows), generating an electric counterflow (white dashed arrows). Because of the bosonic nature of the excitons, the exciton thermocouple can have a higher figure of merit than the traditional thermocouple of Fig. 1.

temperature, enhances the electric counterflow conductivity and gives rise to a high exciton thermal conductivity which reduces the effect of the phonon thermal conductivity $\kappa_{\mathrm{ph}}$.

These are the two proposed pillars of bilayer-exciton thermoelectrics: better thermopower and better conductivities. We suggest two possible material choices for the bilayer-exciton thermocouple: a Bi-based system of $p$ - and $n$-doped $\mathrm{Bi}_{2} \mathrm{Te}_{3}$ and an oxide system consisting of $n$-doped $\mathrm{SrTiO}_{3}$ and $p$-type $\mathrm{Ca}_{3} \mathrm{Co}_{4} \mathrm{O}_{9}$. The expected

TABLE I. Room-temperature properties of traditional thermoelectrics compared to bilayer-exciton systems. Here we compare three traditional thermoelectrics (doped $\mathrm{Bi}_{2} \mathrm{Te}_{3}$ [3,16], Nb-doped $\mathrm{SrTiO}_{3}$ [31-33], and $\mathrm{Ca}_{3} \mathrm{Co}_{4} \mathrm{O}_{9}$ [31-33]) with two bilayerexciton thermocouples based on known experimental data. The Bi-based exciton bilayer consists of $p$ - and $n$-doped $\mathrm{Bi}_{2} \mathrm{Te}_{3}$; the oxide-based exciton bilayer is a $\mathrm{SrTiO}_{3} / \mathrm{Ca}_{3} \mathrm{Co}_{4} \mathrm{O}_{9}$ heterostructure. The columns display the optimal carrier density $n\left(\mathrm{~cm}^{-3}\right)$, degeneracies $g_{s} g_{0}$, thermopower $\alpha\left(\mu \mathrm{V} \mathrm{K}^{-1}\right)$, phonon thermal conductivity $\kappa_{\mathrm{ph}}\left(\mathrm{Wm}^{-1} \mathrm{~K}^{-1}\right)$, electron (exciton) thermal conductivity $\kappa_{e}\left(\kappa_{\mathrm{ex}}\right)\left(\mathrm{Wm}^{-1} \mathrm{~K}^{-1}\right)$, and the figure of merit $z T$. For bilayer excitons, the $\kappa_{\mathrm{ph}}$ is taking into account the contribution of phonons in both layers. Clearly, the exciton systems exhibit a tremendous enhancement of the thermoelectric figure of merit $z T$.

\begin{tabular}{lccrrrc}
\hline \hline Materials & $n$ & $g_{s} g_{0}$ & \multicolumn{1}{c}{$\alpha$} & \multicolumn{1}{c}{$\kappa_{\mathrm{ph}}$} & $\kappa_{e}\left(\kappa_{\mathrm{ex}}\right)$ & $z T$ \\
\hline Doped $\mathrm{Bi}_{2} \mathrm{Te}_{3}$ & $10^{19}$ & $2 \times 6$ & 220 & 0.8 & 0.8 & 1.0 \\
Bi-based exciton & $3 \times 10^{18}$ & $4 \times 36$ & 1060 & 1.6 & 7.0 & 63 \\
$\mathrm{Nb}^{-\mathrm{SrTiO}_{3}}$ & $6 \times 10^{20}$ & $2 \times 3$ & 150 & 8.2 & 0.9 & 0.08 \\
$\mathrm{Ca}_{3} \mathrm{Co}_{4} \mathrm{O}_{9}$ & $1.9 \times 10^{21}$ & 2 & 120 & 3.0 & 0.5 & 0.08 \\
O-based exciton & $1.0 \times 10^{21}$ & $4 \times 3$ & 350 & 11.2 & 8.3 & 3.6 \\
\hline \hline
\end{tabular}


enhancements of thermopower and thermal conductivities are shown in Table I, which comprises our main result. In the remainder of this paper, we first derive the relations between the exciton correlation functions and the counterflow transport coefficients, in Sec. II. Subsequently, we will describe the enhancement of the thermopower in Sec. III and the conductivity in Sec. IV. We end in Sec. V with suggestions towards experimental realization of a bilayerexciton thermocouple.

\section{EXCITON TRANSPORT AND COUNTERFLOW COEFFICIENTS}

Since an exciton is the bound state of an electron and a hole, naively one would expect that excitons would not display any electrical effects. However, in the counterflow setup, the spatial separation of the electrons and holes of the bilayer exciton allows for an electric response [28,29]. In this section, we discuss how bilayer-exciton response functions can be used to express the figure of merit for a bilayer-exciton thermocouple.

We consider the limit where the Coulomb attraction between electrons and holes is strong enough to allow us to treat the exciton as a neutral composite particle moving in a 2D layer. The electrons and holes are spatially separated in the direction perpendicular to this layer. In this limit, we envisage a voltage and a temperature gradient as displayed in Fig. 3. By denoting the exciton particle current and heat current as $j_{\mathrm{ex}}$ and $j_{\mathrm{ex} Q}$, respectively, the traditional Onsager relations for this system are $[4,34]$

$$
\begin{aligned}
j_{\mathrm{ex}} & =-\frac{1}{T} L^{(11)} \nabla \bar{\mu}+L^{(12)} \nabla\left(\frac{1}{T}\right), \\
j_{\mathrm{ex} Q} & =-\frac{1}{T} L^{(21)} \nabla \bar{\mu}+L^{(22)} \nabla\left(\frac{1}{T}\right),
\end{aligned}
$$

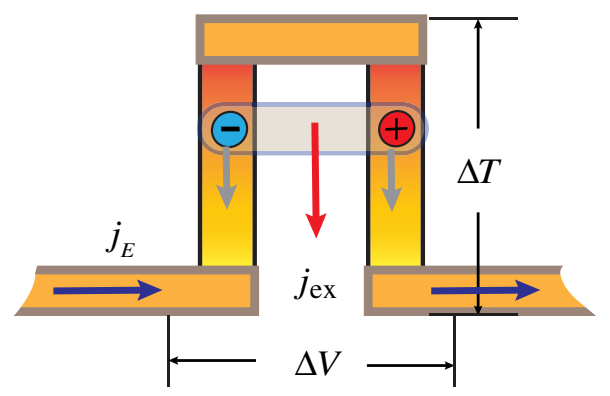

FIG. 3. Thermal and electrical response of strongly bound bilayer excitons. In the counterflow construction, both electrons and holes perceive the same electrical forces (gray arrows) when there is a voltage difference between the external leads. They move together as a composite particle because of the strong exciton binding. The exciton particle current $j_{\mathrm{ex}}$ (red arrow) is driven by both a temperature gradient $\Delta T$ and a voltage $\Delta V$ and generates an electrical current $J_{E}$ (blue arrows), which is proportional to the exciton particle current $J_{E}=e j_{\mathrm{ex}}$. where $\bar{\mu}$ is the effective chemical potential gradient under the voltage difference $\Delta V$ between the external leads. With the length of each lead $l$, we have $\nabla \bar{\mu}=\nabla \mu+2 e E$ and $E=\Delta V / 2 l$. Here the factor 2 comes from both the electron and hole perceiving an electric force $e \Delta V / 2 l$ in the same direction. Thus, effectively the force acting on an exciton equals $e \Delta V / l$. Note that the electric charge $e$ enters in this expression, even though the exciton itself is neutral. This situation is a result of the counterflow setup, where the motion of one bilayer exciton from the top to the bottom of Fig. 3 effectively moves one unit of charge between the leads.

All of the $L^{a b}$ coefficients are directly associated with different exciton current-current correlation functions [34]:

$$
\begin{aligned}
& L^{(11)}=-\frac{1}{\beta} \int_{0}^{\infty} d t e^{-s t} \int_{0}^{\beta} d \beta^{\prime} \operatorname{Tr}\left[\rho_{0} j_{\mathrm{ex}}\left(-t-i \beta^{\prime}\right) j_{\mathrm{ex}}\right], \\
& L^{(12)}=-\frac{1}{\beta} \int_{0}^{\infty} d t e^{-s t} \int_{0}^{\beta} d \beta^{\prime} \operatorname{Tr}\left[\rho_{0} j_{\mathrm{ex}}\left(-t-i \beta^{\prime}\right) j_{\mathrm{ex} Q}\right], \\
& L^{(22)}=-\frac{1}{\beta} \int_{0}^{\infty} d t e^{-s t} \int_{0}^{\beta} d \beta^{\prime} \operatorname{Tr}\left[\rho_{0} j_{\mathrm{ex} Q}\left(-t-i \beta^{\prime}\right) j_{\mathrm{ex} Q}\right] .
\end{aligned}
$$

Finally, when one exciton moves from the hot side to the cold side, there is effectively one unit of charge moved from the left to the right lead. Consequentially, the net electrical current $J_{E}$ between the leads generated by the exciton particle current is given by $J_{E}=e j_{\mathrm{ex}}$. Using this insight, we are now in the position to derive all the relevant transport coefficients. For example, the conductivity $\sigma$ in each leg can found by setting $\Delta \mu=\Delta T=0$ in the Onsager relations, which leads to

$$
\begin{aligned}
J_{E} & =\sigma E, \\
\sigma & =\frac{2 e^{2}}{T} L^{(11)} .
\end{aligned}
$$

However, the counterflow dc conductivity $\sigma_{\mathrm{ex}}$ of the whole systems is only half, since two legs are series connected, which leads to

$$
\sigma_{\mathrm{ex}}=\frac{e^{2}}{T} L^{(11)} .
$$

Similarly, the thermopower and thermal conductivity of the whole thermoelectric couple can be written, respectively, as

$$
\begin{gathered}
\kappa_{\mathrm{ex}}=\frac{1}{T^{2}}\left[L^{(22)}-\frac{\left(L^{(12)}\right)^{2}}{L^{(11)}}\right], \\
\alpha_{\mathrm{ex}}=\frac{1}{e T} \frac{L^{(12)}}{L^{(11)}} .
\end{gathered}
$$

Defining the Lorenz number $L_{\mathrm{ex}}$ for the exciton counterflow system as 


$$
L_{\mathrm{ex}}=\frac{\kappa_{\mathrm{ex}}}{\sigma_{\mathrm{ex}} T},
$$

we can express it in terms of the correlation functions:

$$
L_{\mathrm{ex}}=\frac{1}{e^{2}} \frac{1}{T^{2}}\left[\frac{L^{(22)}}{L^{(11)}}-\left(\frac{L^{(12)}}{L^{(11)}}\right)^{2}\right] .
$$

All correlation functions $L^{(a b)}$ for the excitons can be calculated by using Bose-Einstein statistics, since our excitons are composite bosonic particles in the strong binding limit. The prefactor $1 / e^{2}$ represents that it "effectively" carries charge $e$ due to the magic of the counterflow setup [28]. In this sense, the counterflow transport coefficients of the strongly bound bilayer excitons are equivalent to those of bosonic particles with charge e and mass $2 m$. Consequently, for the whole exciton system, the figure of merit of the counterflow setup can be written as

$$
z T=\frac{\sigma_{\mathrm{ex}} \alpha_{\mathrm{ex}}^{2} T}{\kappa_{\mathrm{ex}}+2 \kappa_{\mathrm{ph}}},
$$

where $2 \kappa_{\mathrm{ph}}$ is the thermal conductivity contribution from phonons in both layers.

In the remainder of this paper, we consider temperatures much higher than the critical temperature $T_{c}$ of the exciton condensate but lower than the exciton binding energy. Additionally, we consider only the case in which the exciton density is low, i.e., the dilute limit, to maximize the thermopower. Thus, within these two limits, the bilayerexciton system approaches the classical limit of bosonic particles, and we can apply the Drude theory for all transport coefficients, which will be done in the next two sections.

\section{ENHANCED THERMOPOWER}

A dominant factor that determines the figure of merit is the thermopower. For Fermi-liquid materials such as metals or degenerate semiconductors, the thermopower Seebeck coefficient $\alpha$ is given by [4]

$$
|\alpha|=\frac{\pi^{2}}{3} \frac{k_{B}}{e}\left(\frac{T}{T_{F}}\right),
$$

where $k_{B}$ is the Boltzmann constant, $e$ is the elementary electric charge, and $T_{F}$ is the Fermi temperature of the material. Therefore, a natural way to increase the thermopower is to reduce the charge carrier density $n$, since $T_{F} \sim n^{2 / 3}$ for a 3D bulk material.

Bilayer excitons, on the other hand, behave like hardcore bosons on a lattice. Following Eq. (8), the counterflow thermopower can be written as

$$
\alpha_{\mathrm{ex}}=\frac{1}{e T} \frac{S^{(12)}}{S^{(11)}}-\frac{\mu}{e T},
$$

where $S^{(11)}=L^{(11)}$ and $S^{(12)}=L^{(12)}-\mu L^{(11)}$ are the exciton correlation functions between the energy current and the exciton particle current, similar to Eq. (4). For temperatures well above the condensation temperature $T_{c}$ and in the dilute limit, the contribution from the correlation function in (13) is minor, and the excitons behave as classical particles, in which case the thermopower is given by the entropy transported per particle divided by the transported charge per particle $[35,36]$.

Recall that, when an exciton is created at the top of the counterflow construction of Fig. 2 and is moved through the bilayer to the bottom, an excess negative charge is created in the left lead and an excess positive charge in the right lead. Effectively, this result implies that a single electric charge has been moved from the left lead to the right lead. Therefore, one moving exciton in the counterflow setup transports one unit of charge.

The entropy of a bilayer exciton is given by the logarithm of the number of possible configurations, whereby spin degeneracy $g_{s}$ and orbital degeneracy $g_{o}$ play an important role $[37,38]$. For excitons on a crystalline lattice with density $\rho$ per unit cell, we find that the excitonic thermopower is given by

$$
\alpha_{\mathrm{ex}}=\frac{k_{B}}{e}\left(\ln g_{s} g_{o}+\ln \frac{1-\rho}{\rho}\right) .
$$

The exciton spin degeneracy is usually $g_{s}=2 \times 2=4$, since both the electron and hole can have two spin states, and the singlet-triplet splitting is negligible compared to the temperature. Similarly, the exciton orbital degeneracy $g_{0}$ is the product of the electron and hole orbital degeneracy, e.g., $6 \times 6$ for doped $\mathrm{Bi}_{2} \mathrm{Te}_{3}$.

In Table I, we compare the Seebeck coefficient of traditional thermoelectric materials to bilayer-exciton systems. The entropic increase of the excitons, as a result of the orbital and spin degeneracies, gives rise to a large thermopower for the bilayer-exciton thermocouple.

Recall that, for most free-electron systems, the Lorenz number is found to be constant [4]. However, in the case of excitons, we no longer have free fermionic electrons, but instead we are dealing effectively with bosonic particles of charge $e$. At high temperatures, the Lorenz number for dilute bosons is given by [39]

$$
L_{\mathrm{ex}}=2\left(\frac{k_{B}}{e}\right)^{2}
$$

which is smaller than the Wiedemann-Franz value by a factor $\frac{L_{\mathrm{WF}}}{L_{\mathrm{ex}}} \approx 1.6$.

\section{ENHANCED CONDUCTIVITY}

Now we discuss the thermal conductivity of excitons. It is known that bulk excitons have an exceptionally large mobility. For example, in cuprous oxide $\mathrm{Cu}_{2} \mathrm{O}$ at low 
temperatures, the exciton mobility is found to be $\mu_{\mathrm{ex}} \sim$ $10^{7} \mathrm{~cm}^{2} / \mathrm{V} \mathrm{s}$ [40], orders of magnitude larger than the mobility of charged carriers in this compound [41]. Measurements on low-temperature bilayer-exciton mobility in GaAs/AlGaAs double quantum wells show similarly high mobilities [42]. Recent results at $T=100 \mathrm{~K}$ in GaAs/AlGaAs double quantum wells suggest that the high mobility of excitons persists up to higher temperatures [43].

Following the sparse experimental results, we can make a qualitative prediction for the high-temperature exciton counterflow conductivity. For any kind of particle, its resistance depends on impurity scattering, scattering with phonons, and particle-particle scattering. In a pure dilute sample at high temperatures, the dominant contribution to the resistivity is given by phonon scattering.

In the case of charged carriers, the scattering with acoustic phonons depends on the deformation potential $D$. Even though excitons are charge neutral, the spatial separation of the electrons and holes still allows for a coupling between the acoustic phonons and the exciton dipole moment. The matrix element between excitons and acoustic phonons is given by a combination of the electron and hole contribution $[22,44]$ :

$$
\sqrt{\frac{2 \hbar k}{M u}}\left[D_{e} \phi_{e}(k)-D_{h} \phi_{h}(k)\right]+O(k),
$$

where $k$ is the phonon wave vector, $u$ is the speed of sound, $M$ is the ion mass, and $D_{e, h}$ are the deformation potentials of the electron and hole, respectively [22,44]. The factors $\phi_{e, h}(k)$ are the wave functions of the electrons and holes that are bound together into the exciton. For a symmetric electron-hole attraction and a simple band structure, these wave functions are of the form $\phi_{e, h}(k)=\left(1+\frac{\mu}{m_{e, h}} \frac{k^{2} a_{\mathrm{ex}}^{2}}{4}\right)^{-2}$, where $\mu=m_{e} m_{h} /\left(m_{e}+m_{h}\right)$ is the reduced mass of the relative electron-hole motion and $a_{\mathrm{ex}}$ is the exciton radius. In the long-wavelength limit, the exciton-phonon coupling becomes independent of the electron and hole masses, and only dependence on the deformation potential remains:

$$
\sqrt{\frac{2 \hbar k}{M u}}\left(D_{e}-D_{h}\right)
$$

The minus sign in Eq. (16) is due to the opposite charge of the electron and hole and, thus, leads to a relatively small exciton-phonon coupling when $D_{e} \approx D_{h}$. Note that Eq. (17) was derived originally for relatively small and thus strongly bound excitons, as, for example, in bulk $\mathrm{Cu}_{2} \mathrm{O}$. There, the radius of a bulk exciton is approximately $7 \AA$; hence, as long as the interlayer excitons are of similar size, we expect the above model to hold.

Thus, if one is able to construct a heterostructure such that the deformation potentials $D_{e, h}$ for the electrons and holes are almost equal, one finds an extremely low excitonphonon scattering rate. In fact, relative to the electronphonon scattering rate,

$$
\frac{\tau_{\mathrm{ex}-\mathrm{ph}}}{\tau_{e-\mathrm{ph}}} \sim\left(\frac{D}{\Delta D}\right)^{2}
$$

where $\Delta D$ is the absolute difference between the electron deformation potential and the hole deformation potential. From the scattering rate $\tau$, the electric counterflow conductivity is given by $\sigma_{\mathrm{ex}}=n e^{2} \tau_{\mathrm{ex}} / 2 m_{\mathrm{ex}}$ with $m_{\mathrm{ex}} \approx 2 m_{e}$, where we assume for simplicity that the electron and hole effective mass are similar. Using the Wiedemann-Franz law, we then find the change in thermal conductivity of the excitons with respect to the electronic thermal conductivity:

$$
\begin{aligned}
& \frac{\sigma_{\mathrm{ex}}}{\sigma_{e}}=\frac{\tau_{\mathrm{ex}-\mathrm{ph}}}{\tau_{e-\mathrm{ph}}} \frac{m_{e}}{m_{\mathrm{ex}}} \sim \frac{1}{2}\left(\frac{D}{\Delta D}\right)^{2}, \\
& \frac{\kappa_{\mathrm{ex}}}{\kappa_{e}}=\frac{\sigma_{\mathrm{ex}}}{\sigma_{e}} \frac{L_{\mathrm{ex}}}{L_{\mathrm{WF}}} \sim 0.3\left(\frac{D}{\Delta D}\right)^{2} .
\end{aligned}
$$

The aforementioned orders-of-magnitude increase of mobility in bulk $\mathrm{Cu}_{2} \mathrm{O}$ [40] suggests that a $10 \%$ difference between the deformation potentials of the electrons and holes is a reasonable assumption. This assumption will lead to a factor of 50 better electric conductivity and a factor of 30 better thermal conductivity of the excitons. We use this estimate of the deformation potentials to obtain the results of Table I.

\section{EXPERIMENTAL REALIZATION}

An idealized exciton thermocouple could therefore have a $z T$ of more than 60 based on current experimental parameters; see Table I. This result is, however, the optimistic theorist perspective. In reality, many pitfalls and engineering problems will lower the figure of merit. Nonetheless, with the ideal $z T \sim 60$, there is much room for shortfalls to still arrive at a sizable figure of merit.

The biggest experimental challenge is to actually make bilayer excitons without condensing them, just like it is difficult to get uncondensed electron-electron pairs. Most material proposals for bilayer-exciton condensation, such as semiconductor quantum wells [24], double-layer graphene [45-49], or topological insulators [50], function in the BCS limit, which means that bilayer excitons are not bound at temperatures above the condensation temperature. Instead, we need materials with strong exciton binding, which can be achieved by bringing the layers close to each other (of the order of several unit cells) to minimize the electron and hole kinetic energy and to ensure that the electrons and holes have similar effective mass and deformation potential. To this end, we propose two possible material choices for the bilayer-exciton thermocouple: bismuth-based bilayers and oxide-based bilayers.

Oxide heterostructures [25-27,51,52], that themselves show good thermoelectric properties [31-33], show a significant enhancement of the figure of merit. Many oxide materials have a layered lattice structure, such as $n$-doped 
$\mathrm{SrTiO}_{3}[18,53]$ and the $p$-type cobaltates $\mathrm{Ca}_{3} \mathrm{Co}_{4} \mathrm{O}_{9}$ and $\mathrm{Na}_{x} \mathrm{CoO}_{2}$ [31]. Techniques such as molecular beam epitaxy (MBE) and pulsed laser deposition make it currently possible to grow heterostructures of layered oxide materials with unit-cell precision. We propose to construct a bilayer system with a few unit-cell layers of Nb-doped $\mathrm{SrTiO}_{3}$, followed by about six unit-cell layers of an oxide insulator like $\mathrm{SrTiO}_{3}$ to avoid recombination of electrons and holes, and then topped a few unit-cell layers of the $p$-type $\mathrm{Ca}_{3} \mathrm{Co}_{4} \mathrm{O}_{9}$. This construction should be repeated in a superlattice structure, with insulating materials between each bilayer, to obtain a material with a bulk thermoelectric effect. By using ion-beam etching, ramp edges can be made, so that one can separately contact each $n$-type and $p$-type layer for the deposition of gold contacts.

We choose these materials because they can be fabricated with similar charge carrier densities of $n \sim 10^{20} \mathrm{~cm}^{-3}$, and the carriers have similar high-temperature electronic thermal conductivities $\kappa_{e} \sim 0.5 \mathrm{~W} \mathrm{~m}^{-1} \mathrm{~K}^{-1}$ [53-55]. $\mathrm{SrTiO}_{3}$ has threefold orbital degeneracy, while the cobaltate is a one-band material. Together with the spin entropy, we expect that at the given densities the exciton thermopower is $\alpha_{\mathrm{ex}} \sim 650 \mu \mathrm{V} \mathrm{K}^{-1}$, which is even a conservative estimate given the $\alpha \sim 1000 \mu \mathrm{V} \mathrm{K}^{-1}$ results in $\mathrm{SrTiO}_{3}$ superlattices [18]. From our earlier analysis, we expect that the exciton thermal conductivity is greatly enhanced. Since the phonon thermal conductivity in oxides is usually the limiting factor in obtaining a high $z T$, the large exciton thermal conductivity yields several-orders-of-magnitude enhancement of $z T$; see Table I. The density dependence of $\alpha, \kappa_{\mathrm{ex}}$, and $z T$ for the oxides is displayed in Fig. 4(a), showing that for a wide window of exciton densities the $\mathrm{SrTiO}_{3} / \mathrm{Ca}_{3} \mathrm{Co}_{4} \mathrm{O}_{9}$ bilayer system has a sizable figure of merit.

The other possibility is to form heterostructures of $p$ - and $n$-doped $\mathrm{Bi}_{2} \mathrm{Te}_{3}$ [16], separated at a distance of approximately $2-3 \mathrm{~nm}$. MBE and chemical vapor deposition are used extensively to fabricate nanostructures of bismuthtellurides [17]. The fact that we have the same material used for both the $p$-type and $n$-type layers implies that the exciton binding and the exciton-phonon coupling are optimal. Together with the sixfold band degeneracy of $\mathrm{Bi}_{2} \mathrm{Te}_{3}$, we find an extraordinary large Seebeck coefficient, $\alpha \sim 1115 \mu \mathrm{V} \mathrm{K}^{-1}$. We thus arrive at an extremely large $z T \sim 60$; see Table I. The density dependence of the thermopower and the thermal conductivity is shown in Fig. 4(b).

A natural question is at which temperatures excitons can still form. Let us therefore make an estimate of the binding energy. Strongly bound bulk excitons can have very large binding energies; for example, the 7- $\AA$ size excitons in $\mathrm{Cu}_{2} \mathrm{O}$ have $V=150 \mathrm{meV}$ [22]. Similarly, the indirect excitons in GaAs/AlGaAs double quantum wells at a distance of approximately $3 \mathrm{~nm}$ have a binding energy of $8 \mathrm{meV}$ [42]. The simplest theoretical estimate,

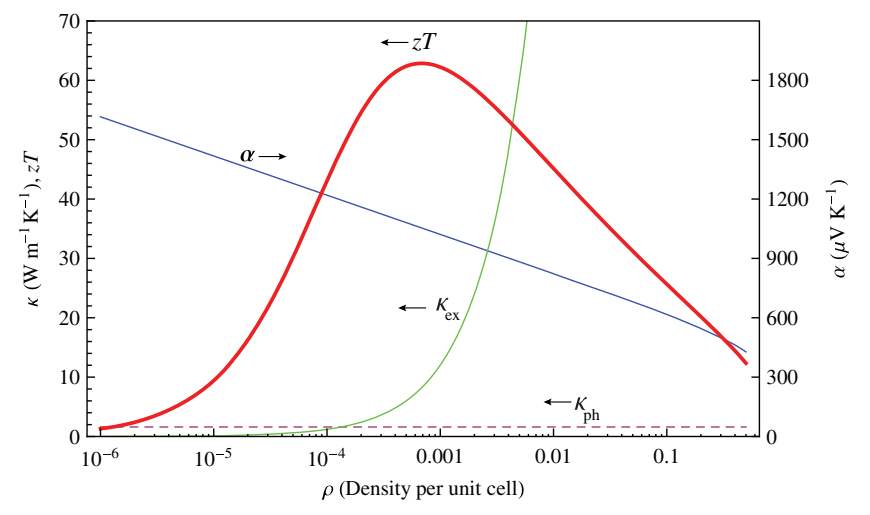

(a)

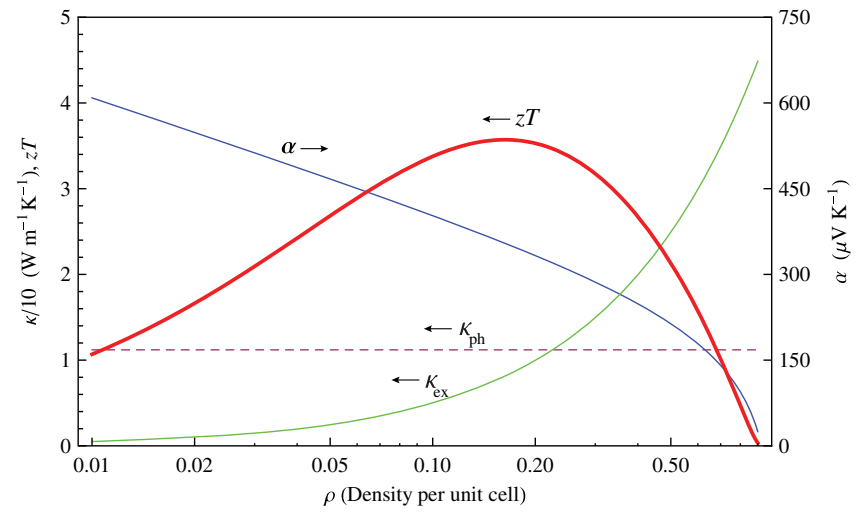

(b)

FIG. 4. Density dependence of the thermoelectric properties. (a) Here we show the figure of merit $z T$, the exciton thermopower $\alpha_{\text {ex }}$, the phonon thermal conductivity $\kappa_{\mathrm{ph}}$, and the exciton thermal conductivity $\kappa_{\mathrm{ex}}$ for the $p$ - and $n$-doped $\mathrm{Bi}_{2} \mathrm{Te}_{3}$ bilayer. The optimal value of $z T$ is used in Table I. (b) The same as (a) but for the bilayer of $n$-doped $\mathrm{SrTiO}_{3}$ and $p$-type $\mathrm{Ca}_{3} \mathrm{Co}_{4} \mathrm{O}_{9}$.

neglecting material complications such as the dielectric mismatch in a heterostructure, is given by the electric energy of an electron and a hole separated at that distance. By using the dielectric constant of GaAs to be $\epsilon \sim 13$, this would give $V \sim 35 \mathrm{meV}$, an estimate roughly 4 times too big. Now consider the oxide materials, having a typical dielectric constant of about $\epsilon \sim 20$. At a distance of $2 \mathrm{~nm}$, the bare electron-hole attraction is $V \sim 35 \mathrm{meV} \approx 400 \mathrm{~K}$; consequently, we expect that in the proposed oxide bilayers the excitons remain bound to at least $100 \mathrm{~K}$.

Nonetheless, at high temperature it is likely that bilayer excitons coexist with unbound electrons and holes. Naturally, this coexistence will reduce the figure of merit, as some of the currents are carried by the less-efficient electrons and holes. A further experimental challenge is therefore to dilute the number of unbound electrons and holes, for example, by bringing the $p$ - and $n$-type materials closer together or changing the insulator layer.

Note that in our current proposal we keep the phonon thermal conductivity constant at its relatively large bulk value, emphasizing only the desired enhancement due to 
the excitonic effects. This fact implies that a further enhancement of the figure of merit can be achieved by combining exciton physics and one of the known techniques to reduce phonon thermal conductivity.

At last, the bilayer-exciton structure not only increases the figure of merit, it also benefits the power factor defined as $\sigma_{\mathrm{ex}} \alpha_{\mathrm{ex}}^{2}$ because of the simultaneous enhancement of both the thermopower and counterflow conductivity. Unlike other strategies of reducing the phonon thermal conductivity, where the power factor is relatively unchanged, the exciton system can increase the power factor 2 orders of magnitude compared to the bulk materials. We acknowledge that a single-bilayer structure may not suitable for technological applications, since it is a two-dimensional thin film with limited output current. However, the repetition of the bilayer into a superlattice structure, as is done in, for example, Ref. [18] for doped $\mathrm{SrTiO}_{3}$ quantum wells, can make the output power macroscopic. The insulating layers staggered between the conducting $n$ - and $p$-type layers in the superlattice structure will reduce the total output power factor, typically of one order of magnitude. Given that each exciton bilayer can have a 2-orders-ofmagnitude enhanced power factor, the final bulk output power factor of the superlattice structure may still be one order of magnitude enhanced.

In conclusion, we show qualitatively that the thermoelectric efficiency of a bilayer-exciton system can be significantly enhanced. We suggest that the nanotechnological engineering of thermoelectric devices should therefore aim at using bilayer excitons, instead of electrons and holes, to generate electricity out of heat.

\section{ACKNOWLEDGMENTS}

We thank Binghai Yan, John Mydosh, Hans Hilgenkamp, Zhi-Xun Shen, Yayu Wang, Yong Xu, Jim Eisenstein, Allan MacDonald, and Aaron Finck for discussions and Jeroen Huijben (Nymus3D) for designing the figures. L. R. is supported by the Netherlands Organisation for Scientific Research (NWO) via a Rubicon grant.

[1] F. J. DiSalvo, Thermoelectric cooling and power generation, Science 285, 703 (1999).

[2] A. F. Ioffe, Semiconductor Thermoelements, and Thermoelectric Cooling (Infosearch, London, 1957).

[3] G. J. Snyder and E. S. Toberer, Complex thermoelectric materials, Nat. Mater. 7, 105 (2008).

[4] N. W. Ashcroft and N.D. Mermin, Solid State Physics (Harcourt College Publications, Orlando, 1976).

[5] A. Sommerfeld, Zur elektronentheorie der metalle, Naturwissenschaften 15, 825 (1927).

[6] A. Sommerfeld, Zur elektronentheorie der metalle auf grund der fermischen statistik, Naturwissenschaften 16, 374 (1928).
[7] L. Lorenz, Ueber das Leitungsvermögen der Metalle für Wärme und Electricität, Ann. Phys. (Berlin) 249, 582 (1881).

[8] L. Lorenz, Ueber das Leitungsvermögen der Metalle für Wärme und Electricität, Ann. Phys. (Berlin) 249, 582 (1881).

[9] G. W. C. Kaye and T. H. Laby, Table of Physical and Chemical Constants (Longmans Green, London, 1996).

[10] G. S. Kumar, G. Prasad, and R. O. Pohl, Experimental determinations of the Lorenz number, J. Mater. Sci. 28, 4261 (1993).

[11] G. A. Slack, in CRC Handbook of Thermoelectrics, edited by D. M. Rowe (CRC Press, Boca Raton, 1995), pp. $407-440$.

[12] T. Takabatake, K. Suekuni, T. Nakayama, and E. Kaneshita, Phonon-glass electron-crystal thermoelectric clathrates: Experiments and theory, Rev. Mod. Phys. 86, 669 (2014).

[13] L. D. Hicks and M. S. Dresselhaus, Effect of quantum-well structures on the thermoelectric figure of merit, Phys. Rev. B 47, 12727 (1993).

[14] Francesco Giazotto, Tero T. Heikkilä, Arttu Luukanen, Alexander M. Savin, and Jukka P. Pekola, Opportunities for mesoscopics in thermometry and refrigeration: Physics and applications, Rev. Mod. Phys. 78, 217 (2006).

[15] L. D. Hicks, T. C. Harman, X. Sun, and M. S. Dresselhaus, Experimental study of the effect of quantum-well structures on the thermoelectric figure of merit, Phys. Rev. B 53, R10493 (1996).

[16] V. L. Kuznetsov, L. A. Kuznetsova, A. E. Kaliazin, and D. M. Rowe, High performance functionally graded and segmented Bi2Te3-based materials for thermoelectric power generation, J. Mater. Sci. 37, 2893 (2002).

[17] R. Venkatasubramanian, E. Siivola, T. Colpitts, and B. O'Quinn, Thin-film thermoelectric devices with high room-temperature figures of merit, Nature (London) 413, 597 (2001).

[18] H. Ohta et al., Giant thermoelectric Seebeck coefficient of a two-dimensional electron gas in $\mathrm{SrTiO}_{3}$, Nat. Mater. 6, 129 (2007).

[19] C. B. Vining, An inconvenient truth about thermoelectrics, Nat. Mater. 8, 83 (2009).

[20] S. I. Shevchenko, Theory of superconductivity of systems with pairing of spatially separated electrons and holes, Fiz. Nizk. Temp. 2, 505 (1976) [Sov. J. Low Temp. Phys. 2, 251 (1976)].

[21] Yu. E. Lozovik and V. I. Yudson, A new mechanism for superconductivity: Pairing between spatially separated electrons and holes, Zh. Eksp. Teor. Fiz. 71, 738 (1976) [Sov. Phys. JETP 44, 389 (1976)].

[22] S. A. Moskalenko and D. W. Snoke, Bose-Einstein Condensation of Excitons and Biexcitons and Coherent Nonlinear Optics with Excitons (Cambridge University Press, Cambridge, England, 2000).

[23] J. P. Eisenstein and A. H. MacDonald, Bose-Einstein condensation of excitons in bilayer electron systems, Nature (London) 432, 691 (2004).

[24] A. A. High, J. R. Leonard, M. Remeika, L. V. Butov, M. Hanson, and A. C. Gossard, Condensation of excitons in a trap, Nano Lett. 12, 2605 (2012). 
[25] L. Rademaker, J. van den Brink, J. Zaanen, and H. Hilgenkamp, Exciton condensation in strongly correlated electron bilayers, Phys. Rev. B 88, 235127 (2013).

[26] L. Rademaker, J. van den Brink, H. Hilgenkamp, and J. Zaanen, Enhancement of spin propagation due to interlayer exciton condensation, Phys. Rev. B 88, 121101(R) (2013).

[27] L. Rademaker, S. Johnston, J. Zaanen, and J. van den Brink, Determinant quantum Monte Carlo study of exciton condensation in the bilayer Hubbard model, Phys. Rev. B 88, 235115 (2013).

[28] J.-J. Su and A.H. MacDonald, How to make a bilayer exciton condensate flow, Nat. Phys. 4, 799 (2008).

[29] A. D. K. Finck, J. P. Eisenstein, L. N. Pfeiffer, and K. W. West, Exciton transport and Andreev reflection in a bilayer quantum Hall system, Phys. Rev. Lett. 106, 236807 (2011).

[30] S. Faniel, E. Tutuc, E. P. De Poortere, C. Gustin, A. Vlad, S. Melinte, M. Shayegan, and V. Bayot, Thermopower of interacting GaAs bilayer hole systems in the reentrant insulating phase near $\nu=1$, Phys. Rev. Lett. 94, 046802 (2005).

[31] J. W. Fergus, Oxide materials for high temperature thermoelectric energy conversion, J. Eur. Ceram. Soc. 32, 525 (2012).

[32] N. V. Nong and N. Pryds, Nanostructured oxide materials and modules for high-temperature power generation from waste heat, Adv. Nat. Sci. Nanosci. Nanotechnol. 4, 023002 (2013).

[33] J. He, Y. Liu, and R. Funahashi, Oxide thermoelectrics: The challenges, progress, and outlook, J. Mater. Res. 26, 1762 (2011).

[34] G. D. Mahan, Many-Particle Physics, 3rd ed. (Kluwer Academic, New York, 2000).

[35] G. Beni, Thermoelectric power of the narrow-band Hubbard chain at arbitrary electron density: Atomic limit, Phys. Rev. B 10, 2186 (1974).

[36] P. M. Chaikin and G. Beni, Thermopower in the correlated hopping regime, Phys. Rev. B 13, 647 (1976).

[37] W. Koshibae and S. Maekawa, Effects of spin and orbital degeneracy on the thermopower of strongly correlated systems, Phys. Rev. Lett. 87, 236603 (2001).

[38] Y. Wang, N. S. Rogado, R. J. Cava, and N. P. Ong, Spin entropy as the likely source of enhanced thermopower in $\mathrm{Na}_{x} \mathrm{Co}_{2} \mathrm{O}_{4}$, Nature (London) 423, 425 (2003).

[39] A. S. Alexandrov and N. F. Mott, Thermal transport in a charged Bose gas and in high- $T_{c}$ oxides, Phys. Rev. Lett. 71, 1075 (1993).

[40] D. P. Trauernicht, J. P. Wolfe, and A. Mysyrowicz, Highly mobile paraexcitons in cuprous oxide, Phys. Rev. Lett. 52, 855 (1984).
[41] Y.S. Lee, M. T. Winkler, S. C. Siah, R. Brandt, and T. Buonassisi, Hall mobility of cuprous oxide thin films deposited by reactive direct-current magnetron sputtering, Appl. Phys. Lett. 98, 192115 (2011).

[42] Z. Vörös, R. Balili, D. W. Snoke, L. Pfeiffer, and K. K. West, Long-distance diffusion of excitons in double quantum well structures, Phys. Rev. Lett. 94, 226401 (2005).

[43] G. Grosso, J. Graves, A. T. Hammack, A. A. High, L. V. Butov, M. Hanson, and A.C. Gossard, Excitonic switches operating at around $100 \mathrm{~K}$, Nat. Photonics 3, 577 (2009).

[44] Y. Toyozawa, Theory of line-shapes of the exciton absorption bands, Prog. Theor. Phys. 20, 53 (1958).

[45] Yu. E. Lozovik and A. A. Sokolik, Electron-hole pair condensation in a graphene bilayer, JETP Lett. 87, 55 (2008).

[46] C.-H. Zhang and Y. N. Joglekar, Excitonic condensation of massless fermions in graphene bilayers, Phys. Rev. B 77, 233405 (2008).

[47] R. Dillenschneider and J. H. Han, Exciton formation in graphene bilayer, Phys. Rev. B 78, 045401 (2008).

[48] H. Min, R. Bistritzer, J.-J. Su, and A. H. MacDonald, Roomtemperature superfluidity in graphene bilayers, Phys. Rev. B 78, 121401(R) (2008).

[49] M. Yu. Kharitonov and K. B. Efetov, Electron screening and excitonic condensation in double-layer graphene systems, Phys. Rev. B 78, 241401(R) (2008).

[50] B. Seradjeh, J. E. Moore, and M. Franz, Exciton condensation and charge fractionalization in a topological insulator film, Phys. Rev. Lett. 103, 066402 (2009).

[51] T. C. Ribeiro, A. Seidel, J. H. Han, and D.-H. Lee, The electronic states of two oppositely doped Mott insulators bilayers, Europhys. Lett. 76, 891 (2006).

[52] A. J. Millis and D. G. Schlom, Electron-hole liquids in transition-metal oxide heterostructures, Phys. Rev. B 82, 073101 (2010).

[53] S. Ohta, T. Nomura, H. Ohta, and K. Koumotoa, Hightemperature carrier transport and thermoelectric properties of heavily La-or $\mathrm{Nb}$-doped $\mathrm{SrTiO}_{3}$ single crystals, J. Appl. Phys. 97, 034106 (2005).

[54] M. Shikano and R. Funahashi, Electrical and thermal properties of single-crystalline $\left(\mathrm{Ca}_{2} \mathrm{CoO}_{3}\right)_{0.7} \mathrm{CoO}_{2}$ with a $\mathrm{Ca}_{3} \mathrm{Co}_{4} \mathrm{O}_{9}$ structure, Appl. Phys. Lett. 82, 1851 (2003).

[55] X. G. Luo, X. H. Chen, G. Y. Wang, C. H. Wang, Y. M. Xiong, H. B. Song, and X. X. Lu, The evolution of magnetotransport properties with carrier concentration in $\mathrm{Ca}_{3} \mathrm{Co}_{4} \mathrm{O}_{9+\delta}$ single crystals, Europhys. Lett. 74, 526 (2006). 